\title{
画HAD
}

ISSN-L: 2530-5115

DOI: http://doi.org/10.22585/hospdomic.v4i1.98

\section{Ventajas de la quimioterapia domiciliaria en los enfermos adultos con neoplasias: revisión sistemática}

Home chemotherapy advantages in adult neoplasia sufferers: a systematic review

Santiago Álvarez Velásquez', Javier Sanz Valero²

1. Universidad Miguel Hernández. Facultad de Farmacia, Campus de Sant Joan d'Alacant. Alicante. España.

2. Universidad Miguel Hernández. Departamento de Salud Pública e Historia de la Ciencia, Campus de Sant Joan d'Alacant. Alicante. España.

Correspondencia/Correspondence

Santiago Álvarez Velásquez

santi2alvarez2@gmail.com

Recibido/Received

13.01 .2020

Aceptado/Accepted

15.01 .2020
Conflicto de Intereses/Competing interest Los autores del trabajo declaran no tener ningún conflicto de interés

CÓMO CITAR ESTE TRABAJO | HOW TO CITE THIS PAPER

Álvarez Velásquez S, Sanz Valero J. Ventajas de la quimioterapia domiciliaria en los enfermos adultos con neoplasias: revisión sistemática. Hosp Domic. 2020;4(1):25-41. 


\section{RESUMEN}

Objetivo: Conocer la evidencia científica con respecto a las posibles ventajas que ofrece la quimioterapia domiciliaria a los enfermos adultos con neoplasias.

Método: Revisión crítica y sistemática. Los datos se obtuvieron de la consulta a las siguientes bases de datos bibliográficas: MEDLINE (vía PubMed), Cochrane Library, Embase, Scopus y Web of Science. Los términos utilizados, como descriptores y como texto en los campos de registro del título y el resumen, fueron "Home Care Services", "Drug Therapy" y "Neoplasms", utilizando los filtros «Humans», "Adult: 19+ years» y «Clinical Trial». Fecha de la búsqueda octubre de 2019. La calidad documental de los artículos se evaluó mediante el cuestionario CONSORT.

Resultados: De las 790 referencias recuperadas, tras depurar las repeticiones y aplicar los criterios de inclusión y exclusión, se seleccionaron 15 ensayos clínicos. En 12 (80\%) de los ensayos revisados se observó que la quimioterapia administrada en el hogar fue segura, factible, beneficiosa y predominantemente preferida por los pacientes. Con respecto al coste del tratamiento se comprobó discrepancias entre algunos trabajos, donde 3 (20\%) estudios señalaban un mayor coste del tratamiento domiciliario.

Conclusiones: La quimioterapia domiciliaria es una alternativa factible y segura para los pacientes, la cual es preferida por estos y sus cuidadores. El mayor o menor coste dependió principalmente del medicamento y tipo de tratamiento.

Palabras clave: Quimioterapia; Antineoplásicos; Citostáticos; Servicios de Atención de Salud a Domicilio; Servicios de Atención a Domicilio Provisto por Hospital; Cumplimiento y Adherencia al Tratamiento; Satisfacción del Paciente.

\section{ABSTRACT}

Objective: To know the scientific evidence regarding the possible advantages offered by home chemotherapy to adult sufferers with neoplasia.

Method: Critical and systematic review. Data were obtained from the query of the following database: MEDLINE (via PubMed), Cochrane Library, Embase, Scopus and Web of Science. Terms used as descriptors and as text in the title and summary record fields were: "Home Care Services", "Drug Therapy" and "Neoplasms", using the filters "Humans», "Adult: 19+ years" and «Clinical Trial». Searching date October 2019. The documental quality of the articles was evaluated by using CONSORT questionnaire.

Results: From 790 recovered references, after refining the repetitions and applying the inclusion and exclusion criteria, 15 clinical trials were selected. In 12 (80\%) of the revised trials it was observed that chemotherapy administrated at home was safe, feasible, beneficial and predominantly preferred by the patients. Regarding the cost of treatment, discrepancies were found between some trials, where 3 studies (20\%) indicated a higher cost of home treatment.

Conclusions: Home chemotherapy is a feasible and safe alternative for patients, which is preferred by them and their caregivers. The higher or lower cost depended mainly on the medication and type of treatment.

Keywords: Drug Therapy; Antineoplastic Agents; Cytostatic Agents; Home Care Services; Home Care Services, Hospital-Based; Treatment Adherence and Compliance; Patient Satisfaction. 


\section{INTRODUCCIÓN}

Hoy en día, la hospitalización a domicilio ( $\mathrm{HaD}$ ) aporta ventajas como alternativa a la hospitalización convencional. Al mismo tiempo, existe evidencia acerca de dichas ventajas en lo que respecta a la eficacia, seguridad y al alto nivel de satisfacción de esta modalidad asistencial. Los factores clave para su correcta implementación son garantizar una adecuada selección de los pacientes dependientes de $\mathrm{HaD}$ y disponer de un equipo experimentado en atención de casos complejos en el domicilio (1).

Así, la HaD se define como un servicio que proporciona tratamiento activo por profesionales de la salud en el hogar del paciente, por una afección que de otro modo requeriría atención hospitalaria (2). Por otra parte, el desarrollo de los servicios hospitalarios en el hogar tiene también como objetivo mejorar la percepción del paciente respecto al tratamiento y mejorar su calidad de vida al ofrecer la posibilidad de dicha atención en un ambiente amigable y con la misma eficiencia en la atención médica (3).

En esta línea, hasta hace unos años el tratamiento farmacológico contra la neoplasia se había administrado tradicionalmente en unidades especializadas dentro de las instalaciones hospitalarias. Pero, los avances tecnológicos y la mejora en la calidad de la atención han hecho posible que, hoy en día, la mayoría de la quimioterapia ya se pueda administrar de manera ambulatoria (4).

La enfermedad neoplásica provoca cambios físicos, emocionales y sociales que generan diversas necesidades en los pacientes y sus familias. La menor estancia hospitalaria, la mayor sobrevida y el amplio número de pacientes que se mantienen en sus hogares recibiendo cuidado, han creado una mayor necesidad de asistencia en aspectos como el transporte de los pacientes, el cuidado en casa, el desarrollo de las actividades de la vida diaria y en la forma de reducir los costos que la enfermedad genera (5). En este sentido, es interesante estudiar el tratamiento de la neoplasia en el domicilio, ya que los esquemas asistenciales para esta patología se desarrollaron rápidamente (3).

La HaD del siglo XXI ya no se plantea exclusivamente como alternativa asistencial, se posiciona como la mejor alternativa para numerosos procesos médicos y quirúrgicos en un determinado momento evolutivo de los mismos. Y esto es posible porque la $\mathrm{HaD}$ proporciona intensidad de cuidados mediante una atención multidisciplinar centrada en el paciente, humanizando esta atención e integrando al paciente y su familia en los cuidados. Fomenta la continuidad asistencial y es segura, efectiva, eficiente y satisfactoria (6). Además, a pesar de que no se dispone de estudios exhaustivos que lo confirmen, distintos trabajos en la literatura internacional y un número mayor de grupos nacionales que aportan sus datos de costes, parecen corroborar que a los beneficios asistenciales hay que sumar un ahorro de costes respecto a la hospitalización convencional (1).

En consecuencia, el objetivo de esta revisión fue conocer la evidencia científica con respecto a las posibles ventajas que ofrece la quimioterapia domiciliaria a los enfermos adultos con neoplasias.

\section{MATERIAL Y MÉTODO}

\section{Diseño}

Estudio descriptivo transversal y análisis crítico de los trabajos recuperados mediante revisión sistemática. 


\section{Fuente de obtención de los datos}

Los datos se obtuvieron de la consulta directa y acceso, vía Internet, a las siguientes bases de datos bibliográficas del ámbito de las ciencias de la salud: MEDLINE (vía PubMed), Cochrane Library, Embase, Scopus y Web of Science.

\section{Tratamiento de la información}

Para definir los términos de la búsqueda se consultó el Thesaurus desarrollado por la U.S. National Library of Medicine. Se consideró adecuado el uso de los términos "Home Care Services", "Drug Therapy" y "Neoplasms", como descriptores y como texto en los campos de registro del título y el resumen, conformando la sintaxis de búsqueda final mediante la intersección booleana de tres ecuaciones: (Población) AND (Intervención) AND (Resultado).

Población - Personas adultas atendidas por los servicios de cuidado domiciliario:

"Home Care Services"[MeSH] OR "Home Care"[Title/Abstract] OR "Domiciliary Care"[Title/Abstract] OR "Home Care Services, Hospital- Based"[Mesh] OR "Hospital-Based Home Care"[All Fields] OR "Hospital Based Home Care"[Title/Abstract] OR "Hospital Home Care Services"[Title/Abstract] OR "Hospital-Based Home Care Services"[Title/Abstract] OR "Hospital Based Home Care Services"[Title/Abstract] OR "Home Hospitalization"[Title/Abstract] OR "Hospital at Home"[Title/Abstract] OR "Hospitalat-Home"[Title/Abstract] OR "Hospital Home Care"[Title/Abstract] OR "Hospital at Home Care"[Title/Abstract] OR "Hospital in the Home"[Title/Abstract]

Intervención - Tratamiento quimioterápico:

"Drug Therapy"[Mesh] OR "Drug Therapy"[Title/Abstract] OR "Drug Therapies"[Title/ Abstract] OR "Chemotherapy"[Title/Abstract] OR "Chemotherapies"[Title/Abstract] OR "Pharmacotherapy"[Title/Abstract] OR "Pharmacotherapies"[Title/Abstract] OR "Cytostatic Agents"[Mesh] OR "Cytostatic Agents"[Title/Abstract] OR "Cytostatics"[Title/Abstract] OR "Cytostatic Drugs"[Title/Abstract] OR "Antineoplastic Drugs"[Title/Abstract] OR "Antineoplastics"[Title/Abstract] OR "Chemotherapeutic Anticancer Drug"[Title/Abstract] OR "Antitumor Drugs"[Title/Abstract] OR "Cancer Chemotherapy Agents"[Title/ Abstract] OR "Cancer Chemotherapy Drugs"[Title/Abstract] OR "Chemotherapeutic Anticancer Agents"[Title/Abstract] OR "Anticancer Agents"[Title/Abstract] OR "Antitumor Agents"[Title/Abstract] OR "Antineoplastic Agents/drug therapy"[Mesh]

Resultado - Neoplasias:

"Neoplasms"[Mesh] OR "Neoplasms"[Title/Abstract] OR "Neoplasia"[Title/Abstract] OR "Neoplasm"[Title/Abstract] OR "Tumor"[Title/Abstract] OR "Cancer"[Title/Abstract] OR "Malignancy"[Title/Abstract] OR "Malignancies"[Title/Abstract] OR "Malignant Neoplasm"[Title/Abstract]

La ecuación de búsqueda final se desarrolló para su empleo en la base de datos MEDLINE, vía PubMed, utilizando los filtros: «Humans», «Adult: 19+ years» y «Clinical Trial».

Esta estrategia se adaptó a las características de cada una del resto de bases de datos consultadas. La búsqueda se realizó desde la primera fecha disponible, de acuerdo a las características de cada base de datos, hasta octubre de 2019 y se completó con el examen del listado bibliográfico de los artículos que fueron seleccionados. 


\section{Selección final de los artículos}

Se escogieron para la revisión y análisis crítico los artículos que cumplieron los siguientes criterios: adecuarse a los objetivos de la búsqueda (quimioterapia en el domicilio), estar publicados en revistas arbitradas por pares y escritos en inglés, español o portugués.

La selección de los artículos pertinentes se realizó de forma independiente por los autores: SAV y JSV. Para dar por válida la inclusión de los estudios se estableció que la valoración de la concordancia entre estos autores (índice Kappa) debía ser superior al 60\% (fuerza de la concordancia buena). Siempre que se cumpliera esta condición, las posibles discordancias se solucionaron mediante el consenso entre los autores (7).

Para valorar la calidad de los documentos seleccionados se utilizaron las directrices para la comunicación de los ensayos clínicos CONSORT (CONsolidated Standards of Reporting Trials) (8), que contiene un listado de 25 aspectos esenciales que deben describirse en la publicación de estos estudios. Para cada artículo seleccionado se asignó un punto por cada ítem presente (en caso de no ser aplicable no puntuaba). Cuando un ítem estaba compuesto por varios puntos, estos se evaluaron de forma independiente, dándole el mismo valor a cada uno de ellos y posteriormente se realizó un promedio (siendo éste el resultado final de ese ítem), de tal forma que en ningún caso se pudiera superar la puntuación de un punto por ítem.

\section{Extracción de los datos}

El control de la corrección de los datos se realizó mediante dobles tablas que permitieron la detección de las desviaciones y su subsanación mediante nueva consulta de los originales.

Para determinar la actualidad de los artículos se calculó el semiperíodo de Burton-Kebler (la mediana de la edad) y el Índice de Price (porcentaje de artículo con edad inferior a los 5 años).

Los estudios se agruparon según las variables a estudio, con el fin de sistematizar y facilitar la comprensión de los resultados, considerando los siguientes datos: primer autor y año de publicación, tipo de estudio, país, población incluida en los artículos revisados, patología de la población, intervención (tratamiento administrado), periodo del tratamiento, resultados obtenido de la intervención.

\section{RESULTADOS}

Al aplicar los criterios de búsqueda descritos se recuperaron un total de 790 referencias: 366 (46,3\%) en Scopus, 138 (17,5\%) en Embase, 128 (16,2\%) en Cochrane Library, 106 (13,4\%) en Web of Science y 52 (6,6\%) en MEDLINE (vía PubMed).

Tras depurar los $211(26,7 \%)$ registros repetidos, aplicar los criterios de inclusión y exclusión, y consultar los listados bibliográficos de los artículos seleccionados (figura 1), fue posible seleccionar 15 documentos (9-23) para su revisión y análisis crítico (tabla 1).

El acuerdo sobre la pertinencia de los estudios seleccionados, entre los dos evaluadores, calculado mediante el índice Kappa fue del 100\%.

Los artículos elegidos presentaron una obsolescencia, según el Índice de Burton Kebler, igual a 17 años, con un Índice de Price del 13,3\%. El año con mayor número de trabajos publicados fue el 2000, con tres publicaciones (18-20). 
Figura 1 - Identificación y selección de estudios
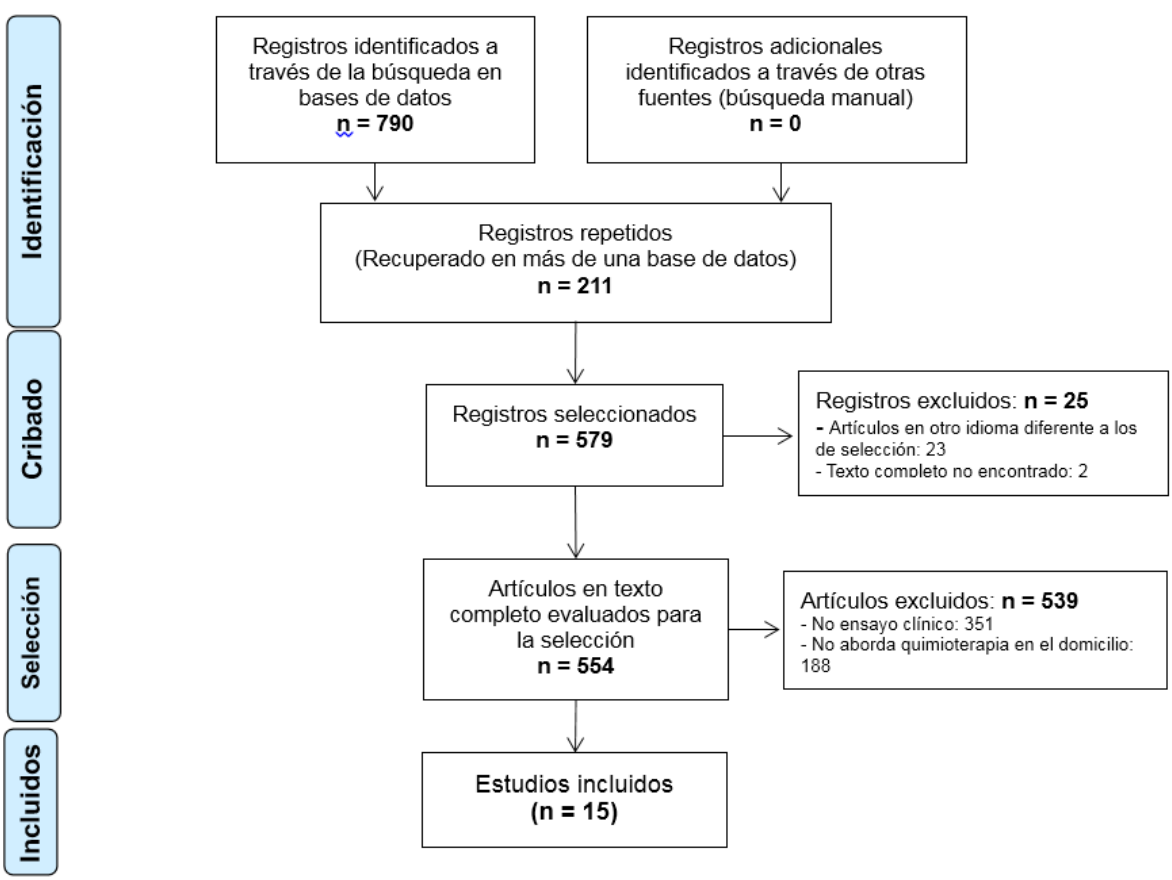

Al evaluar la calidad de los artículos seleccionados para la revisión, mediante el cuestionario CONSORT, las puntuaciones oscilaron entre mínimo de 11 (44\% de cumplimiento) y máximo de 19,50 (78\% de cumplimiento), con mediana igual a 13,5 (tabla 2).

Los documentos revisados se publicaron en diferentes países, siendo Alemania el país que aportó un mayor número de referencias, con $4(26,7 \%)$ estudios en total $(15,16,20,23)$. Además, los 15 (100\%) artículos incluidos en la tabla de la revisión estaban redactados en inglés (9-23).

El estudio de Atzpodien et al. (16), de 2002, presentó la población con el mínimo de edad, 57 años, y el de Tassinari et al. (13), de 2007, incluyó la población con la edad máxima de 72 años.

La patología estudiada en todos los estudios fue la neoplasia, siendo la más prevalente la metastásica $(13,19,20,23)$.

El periodo de tratamiento de los documentos revisados presentó un mínimo de 21 días (22) y un máximo de 21 meses (16). 
Tabla 1. Características de los estudios incluidos en la revisión sobre ventajas de la quimioterapia domiciliaria en los enfermos adultos con neoplasias

\begin{tabular}{|c|c|c|c|c|c|c|c|}
\hline Autor; año & Diseño & País & Población & $\begin{array}{l}\text { Patología } \\
\text { (neoplasia) }\end{array}$ & $\begin{array}{l}\text { Intervención } \\
\text { (tratamiento) }\end{array}$ & $\begin{array}{l}\text { Periodo del } \\
\text { tratamiento }\end{array}$ & $\begin{array}{l}\text { Resultados } \\
\text { obtenidos }\end{array}$ \\
\hline $\begin{array}{l}\text { Larsen, et al. } \\
2018 \text { (9) }\end{array}$ & $\begin{array}{l}\text { Ensayo } \\
\text { clínico }\end{array}$ & Dinamarca & $\begin{array}{l}\mathrm{N}=42 \\
\text { Grupo } \mathrm{A}=21 \\
\text { Edad media } 64 \\
\text { años } \\
\mathrm{H} / \mathrm{M}=14 / 7 \\
\text { Grupo } \mathrm{B}=21 \\
\text { Edad media } 67 \\
\text { años } \\
\mathrm{H} / \mathrm{M}=10 / 11\end{array}$ & $\begin{array}{l}\text { Neoplasia } \\
\text { de colon }\end{array}$ & $\begin{array}{l}\text { Primer ciclo de } \\
\text { ambos grupos } \\
\text { en clínica } \\
\text { ambulatoria. } \\
\text { Grupo } \mathrm{A}=4 \text { ciclos } \\
\text { (capecitabina y } \\
\text { oxaliplatino) en el } \\
\text { hogar y } 3 \text { ciclos en } \\
\text { la clínica. } \\
\text { Grupo } \mathrm{B}=3 \text { ciclos } \\
\text { en el hogar y } 4 \\
\text { ciclos en la clínica. }\end{array}$ & 6 meses & $\begin{array}{l}\text { El tratamiento fue } \\
\text { factible, seguro } \\
\text { y los pacientes } \\
\text { se sintieron } \\
\text { cómodos con } \\
\text { la quimioterapia } \\
\text { domiciliaria. Y, la } \\
\text { consideraron una } \\
\text { alternativa valiosa } \\
\text { frente al ingreso } \\
\text { hospitalario. }\end{array}$ \\
\hline $\begin{array}{l}\text { Lal, et al. } \\
2015 \text { (10) }\end{array}$ & $\begin{array}{l}\text { Ensayo } \\
\text { clínico } \\
\text { fase II }\end{array}$ & $\begin{array}{l}\text { Reino } \\
\text { Unido y } \\
\text { Suecia }\end{array}$ & $\begin{array}{l}\mathrm{N}=52 \text { (primer ciclo } \\
\text { en hospital) } \\
\text { Edad media } 66 \\
\text { años } \\
\mathrm{H} / \mathrm{M}=26 / 26\end{array}$ & $\begin{array}{l}\text { Neoplasia } \\
\text { avanzada } \\
\text { de } \\
\text { pulmón no } \\
\text { escamoso } \\
\text { de } \\
\text { células no } \\
\text { pequeñas }\end{array}$ & $\begin{array}{l}\text { Pemetrexed en } \\
\text { casa tras una } \\
\text { primera dosis en } \\
\text { hospital. } \\
47 \text { pacientes al } \\
\text { menos } 1 \text { ciclo en } \\
\text { casa. } \\
7 \text { pacientes no } \\
\text { continuaron } \\
\text { el ciclo por } \\
\text { reacciones } \\
\text { adversas (1 } \\
\text { prefirió hacerlo en } \\
\text { hospital). }\end{array}$ & $\begin{array}{l}\text { La media de } \\
\text { la terapia de } \\
\text { mantenimiento } \\
\text { fue de } 8,7 \\
\text { meses }\end{array}$ & $\begin{array}{l}\text { El mantenimiento } \\
\text { de Pemetrexed en } \\
\text { estos pacientes } \\
\text { mostró buenos } \\
\text { resultados en } \\
\text { cumplimiento } \\
\text { y viabilidad. } \\
\text { Quimioterapia } \\
\text { en el domicilio } \\
\text { preferida por los } \\
\text { pacientes frente a } \\
\text { otro servicio. }\end{array}$ \\
\hline $\begin{array}{l}\text { Lal, et al. } \\
2013 \text { (11) }\end{array}$ & $\begin{array}{l}\text { Ensayo } \\
\text { clínico } \\
\text { fase II }\end{array}$ & $\begin{array}{l}\text { Reino } \\
\text { Unido y } \\
\text { Suecia }\end{array}$ & $\begin{array}{l}\mathrm{N}=50 \\
\text { Edad: No consta } \\
\text { H/M: No consta }\end{array}$ & $\begin{array}{l}\text { Neoplasia } \\
\text { avanzada } \\
\text { de } \\
\text { pulmón no } \\
\text { escamoso } \\
\text { de } \\
\text { células no } \\
\text { pequeñas }\end{array}$ & $\begin{array}{l}\text { Pemetrexed } 3 \\
\text { semanas hasta } \\
\text { enfermedad } \\
\text { progresiva, } \\
\text { toxicidad } \\
\text { inaceptable o } \\
\text { abandono por } \\
\text { algún otro motivo. }\end{array}$ & 21 días & $\begin{array}{l}\text { Pacientes y } \\
\text { cuidadores } \\
\text { informaron } \\
\text { positivamente } \\
\text { sobre la } \\
\text { administración } \\
\text { domiciliaria y } \\
\text { la prefirieron a } \\
\text { la hospitalaria } \\
\text { (menos un } \\
\text { paciente, } \\
\text { por efectos } \\
\text { secundarios). }\end{array}$ \\
\hline
\end{tabular}


Tabla 1. Características de los estudios incluidos en la revisión sobre ventajas de la quimioterapia domiciliaria en los enfermos adultos con neoplasias

\begin{tabular}{|c|c|c|c|c|c|c|c|}
\hline Autor; año & Diseño & País & Población & $\begin{array}{l}\text { Patología } \\
\text { (neoplasia) }\end{array}$ & $\begin{array}{l}\text { Intervención } \\
\text { (tratamiento) }\end{array}$ & $\begin{array}{l}\text { Periodo del } \\
\text { tratamiento }\end{array}$ & $\begin{array}{l}\text { Resultados } \\
\text { obtenidos }\end{array}$ \\
\hline $\begin{array}{l}\text { Beijer, et al. } \\
2007 \text { (12) }\end{array}$ & $\begin{array}{l}\text { Ensayo } \\
\text { aleatorizado } \\
\text { controlado }\end{array}$ & Holanda & $\begin{array}{l}\mathrm{N}=51 \\
\text { Edad media } 68,1 \\
\text { años } \\
\mathrm{H} / \mathrm{M}=35 / 16 \\
\text { Gcontrol = } 27 \mathrm{No} \\
\text { completaron las } \\
8 \text { infusiones o } \\
\text { murieron } \\
\text { GATP = } 24 \text { sí } \\
\text { completaron las } 8 \\
\text { infusiones (además } \\
12 \text { lo continuaron) }\end{array}$ & $\begin{array}{l}\text { Neoplasia } \\
\text { con } \\
\text { esperanza } \\
\text { de vida } \\
\text { inferior } 6 \\
\text { meses }\end{array}$ & $\begin{array}{l}\text { Infusiones } \\
\text { intravenosas de } \\
\text { Adenosín trifosfato } \\
\text { (ATP) en el hogar }\end{array}$ & 8 semanas & $\begin{array}{l}\text { La administración } \\
\text { de ATP en casa } \\
\text { fue segura y la } \\
\text { mayoría ( } 63 \% \text { ) } \\
\text { no presentaron } \\
\text { efectos } \\
\text { secundarios. }\end{array}$ \\
\hline $\begin{array}{l}\text { Tassinari, } \\
\text { et al. } \\
2007 \text { (13) }\end{array}$ & $\begin{array}{l}\text { Ensayo } \\
\text { clínico }\end{array}$ & Italia & $\begin{array}{l}\mathrm{N}=42 \\
\text { No constan grupos. } \\
\text { Edad media } 72 \\
\text { años } \\
\mathrm{H} / \mathrm{M}=15 / 27\end{array}$ & $\begin{array}{l}\text { Metástasis } \\
\text { ósea }\end{array}$ & $\begin{array}{l}\text { Tratamiento en el } \\
\text { hogar con ácido } \\
\text { zoledrónico }\end{array}$ & 130 días & $\begin{array}{l}\text { El tratamiento fue } \\
\text { seguro y se pudo } \\
\text { llevar a cabo en el } \\
\text { hogar. }\end{array}$ \\
\hline $\begin{array}{l}\text { Smith, et al. } \\
2004(14)\end{array}$ & $\begin{array}{l}\text { Ensayo } \\
\text { clínico, } \\
\text { controlado } \\
\text { aleatorizado }\end{array}$ & $\begin{array}{l}\text { Reino } \\
\text { Unido }\end{array}$ & $\begin{array}{l}\mathrm{N}=37 \\
\text { Edad media } 64,8 \\
\text { años } \\
\mathrm{H} / \mathrm{M}=19 / 18 \\
\mathrm{G} 1=19, \\
\text { primero atención } \\
\text { domiciliaria, luego } \\
\text { hospital. } \\
\mathrm{G} 2=18, \\
\text { primero hospital } \\
\text { luego atención } \\
\text { domiciliaria }\end{array}$ & $\begin{array}{l}\text { Mieloma } \\
\text { múltiple }\end{array}$ & $\begin{array}{l}\text { Tratamiento con } \\
\text { pamidronato cada } \\
4 \text { semanas durante } \\
3 \text { meses en el } \\
\text { hogar y } 3 \text { meses } \\
\text { en el hospital. } \\
\text { Bifosfonato como } \\
\text { terapia profiláctica } \\
\text { y de rutina. }\end{array}$ & 6 meses & $\begin{array}{l}\text { Fue seguro } \\
\text { y práctico } \\
\text { administrar } \\
\text { pamidronato en el } \\
\text { hogar. La mayoría } \\
\text { de los pacientes } \\
\text { lo prefirieron al } \\
\text { tratamiento en el } \\
\text { hospital }\end{array}$ \\
\hline $\begin{array}{l}\text { Atzpodien, } \\
\text { et al. } \\
2003 \text { (15) }\end{array}$ & $\begin{array}{l}\text { Ensayo } \\
\text { clínico }\end{array}$ & Alemania & $\begin{array}{l}\mathrm{N}=22 \\
\text { No constan grupos. } \\
\mathrm{H} / \mathrm{M}=16 / 6 \\
\text { Edad media } 64 \\
\text { años }\end{array}$ & $\begin{array}{l}\text { Neoplasia } \\
\text { renal }\end{array}$ & $\begin{array}{l}\text { Tratamiento } \\
\text { en casa con } \\
\text { Interleukina } 2 \text { e } \\
\text { interferón alfa }\end{array}$ & 8 semanas & $\begin{array}{l}\text { No se encontraron } \\
\text { diferencias entre } \\
\text { el tratamiento en } \\
\text { el hospital o en } \\
\text { casa }\end{array}$ \\
\hline
\end{tabular}




\begin{tabular}{|c|c|c|c|c|c|c|c|}
\hline Autor; año & Diseño & País & Población & $\begin{array}{l}\text { Patología } \\
\text { (neoplasia) }\end{array}$ & $\begin{array}{l}\text { Intervención } \\
\text { (tratamiento) }\end{array}$ & $\begin{array}{l}\text { Periodo del } \\
\text { tratamiento }\end{array}$ & $\begin{array}{l}\text { Resultados } \\
\text { obtenidos }\end{array}$ \\
\hline $\begin{array}{l}\text { Atzpodien, } \\
\text { et al. } \\
2002 \text { (16) }\end{array}$ & $\begin{array}{l}\text { Ensayo } \\
\text { clínico }\end{array}$ & Alemania & $\begin{array}{l}\mathrm{N}=443 \\
-\mathrm{IL}-2^{*} / \mathrm{IFN}-\mathrm{a}^{*}: 97 \\
\mathrm{H} / \mathrm{M}=69 / 38 \\
\text { Edad media } 58 \\
\text { años } \\
\text { - IL-2/IFN-a/5-FU*: } \\
260 \\
\text { H/M=188/72 } \\
\text { Edad media } 58 \\
\text { años } \\
\text { - IL-2/IFN-a/5-FU } \\
/ 13 c R A: 86 \\
\text { H/M=66/20 } \\
\text { Edad media } 57 \\
\text { años }\end{array}$ & $\begin{array}{l}\text { Neoplasia } \\
\text { renal }\end{array}$ & $\begin{array}{l}3 \text { tipos de } \\
\text { tratamiento } \\
\text { llevados a cabo en } \\
\text { el domicilio. } \\
\text { 1: IL-2/IFN-a } \\
\text { 2: IL-2/IFN-a/5-FU } \\
\text { 3: IL-2/IFN-a/5- } \\
\text { FU/13cRA }\end{array}$ & 21 meses & $\begin{array}{l}\text { Los resultados } \\
\text { mostraron un } \\
\text { beneficio a } \\
\text { largo plazo en } \\
\text { pacientes que } \\
\text { recibieron IFN-a e } \\
\text { IL-2 en el domicilio } \\
\text { (beneficio } \\
\text { aumentado } \\
\text { con la adición } \\
\text { intravenosa de } \\
\text { 5-FU y 13cRA } \\
\text { oral). }\end{array}$ \\
\hline $\begin{array}{l}\text { Borras, et al. } \\
2001 \text { (17) }\end{array}$ & $\begin{array}{l}\text { Ensayo } \\
\text { clínico } \\
\text { controlado } \\
\text { aleatorizado }\end{array}$ & España & $\begin{array}{l}\mathrm{N}=87 \\
\mathrm{Gcp^{* }}=45 \\
\text { Edad media } 59,8 \\
\text { años } \\
\mathrm{H} / \mathrm{M}=21 / 24 \\
\mathrm{Ghp}{ }^{*}=42 \\
\text { Edad media } 60,8 \\
\text { años } \\
\mathrm{H} / \mathrm{M}=24 / 18\end{array}$ & $\begin{array}{l}\text { Neoplasia } \\
\text { colorrectal }\end{array}$ & $\begin{array}{l}\text { Tratamiento con } \\
\text { fluorouracilo (con } \\
\text { o sin ácido fólico } \\
\text { o levamisole) en } \\
\text { casa o consulta } \\
\text { externa. }\end{array}$ & 12 meses & $\begin{array}{l}\text { Quimioterapia } \\
\text { domiciliaria fue } \\
\text { una alternativa } \\
\text { aceptable y } \\
\text { segura en esta } \\
\text { patología, que } \\
\text { facilita y mejora } \\
\text { el cumplimiento } \\
\text { terapéutico. }\end{array}$ \\
\hline $\begin{array}{l}\text { Rischin, et } \\
\text { al. } 2000 \text { (18) }\end{array}$ & $\begin{array}{l}\text { Ensayo } \\
\text { clínico } \\
\text { controlado } \\
\text { aleatorizado }\end{array}$ & Australia & $\begin{array}{l}\mathrm{N}=20 \\
\text { Gcp }=11 \text { (5 } \\
\text { ciclofosfamida/ } \\
\text { metotrexato/5-FU } \\
\pm \text { prednisolona, } \\
5 \text { 5-FU } \pm \text { ácido } \\
\text { fólico o levamisol, } 1 \\
\text { CHOP }{ }^{*} \text { ) } \\
\text { Edad media } 59 \\
\text { años } \\
\mathrm{H} / \mathrm{M}=4 / 7 \\
\mathrm{Ghp}=9 \text { (5 } \\
\text { ciclofosfamida/ } \\
\text { metotrexato/5-FU } \\
\pm \text { prednisolona, } 4 \\
5-\mathrm{FU} \pm \text { ácido fólico } \\
\text { O levamisol) } \\
\text { Edad media } 61 \\
\text { años } \\
\mathrm{H} / \mathrm{M}=1 / 8\end{array}$ & $\begin{array}{l}\text { Neoplasia } \\
\text { general }\end{array}$ & $\begin{array}{l}\text { Ciclofosfamida, } \\
\text { metotrexato, } \\
\text { 5-fluorouracilo, } \\
\text { prednisolona, } \\
\text { prednisona, ácido } \\
\text { fólico, levamisol, } \\
\text { doxorrubicina } \\
\text { y/o vincristina; } \\
\text { primero en casa } \\
\text { y luego hospital y } \\
\text { viceversa. }\end{array}$ & 13 meses & $\begin{array}{l}\text { Los pacientes } \\
\text { prefirieron la } \\
\text { quimioterapia } \\
\text { domiciliaria } \\
\text { al tratamiento } \\
\text { hospitalario. } \\
\text { El } 70 \% \text { de los } \\
\text { pacientes hubiera } \\
\text { preferido recibir } \\
\text { los } 2 \text { tratamientos } \\
\text { en el hogar, } \\
\text { mientras que } \\
\text { ninguno prefirió } \\
\text { el tratamiento } \\
\text { completo en el } \\
\text { hospital. } \\
\text { Aumento de } \\
83 \$ \text { por cada } \\
\text { tratamiento de } \\
\text { quimioterapia en } \\
\text { el domicilio. }\end{array}$ \\
\hline
\end{tabular}




\begin{tabular}{|c|c|c|c|c|c|c|c|}
\hline Autor; año & Diseño & País & Población & $\begin{array}{l}\text { Patología } \\
\text { (neoplasia) }\end{array}$ & $\begin{array}{l}\text { Intervención } \\
\text { (tratamiento) }\end{array}$ & $\begin{array}{l}\text { Periodo del } \\
\text { tratamiento }\end{array}$ & $\begin{array}{l}\text { Resultados } \\
\text { obtenidos }\end{array}$ \\
\hline $\begin{array}{l}\text { King, et al. } \\
2000 \text { (19) }\end{array}$ & $\begin{array}{l}\text { Ensayo } \\
\text { clínico }\end{array}$ & Australia & $\begin{array}{l}\mathrm{N}=74 \\
\text { No constan grupos. } \\
\text { No consta } \mathrm{H} / \mathrm{M} \\
\text { No consta edad } \\
\text { media }\end{array}$ & $\begin{array}{l}\text { Neoplasia } \\
\text { de mama } \\
\text { metastásica } \\
\text { o temprana; } \\
\text { Neoplasia } \\
\text { en el cuello } \\
\text { y cabeza. }\end{array}$ & $\begin{array}{l}\text { A) } 5-\text { FU y } \\
\text { levamisol } \\
\text { B) Ciclofosfamida, } \\
\text { metotrexato y } \\
\text { 5-FU (CMF), } \\
\text { intravenoso. } \\
\text { C) Ciclofosfamida, } \\
\text { metotrexato y 5-FU } \\
\text { (CMF), oral. }\end{array}$ & $\begin{array}{l}\mathrm{A}=12 \text { meses } \\
\mathrm{B}=6 \text { ciclos } \\
\text { cada } 21 \text { días } \\
\mathrm{C}=6 \text { ciclos } \\
\text { cada } 28 \text { días }\end{array}$ & $\begin{array}{l}\text { La quimioterapia } \\
\text { en casa resulto } \\
\text { más costosa pero } \\
\text { la mayoría de } \\
\text { los pacientes la } \\
\text { prefirieron frente } \\
\text { al hospital. }\end{array}$ \\
\hline $\begin{array}{l}\text { Oevermann, } \\
\text { et al. } \\
2000(20)\end{array}$ & $\begin{array}{l}\text { Ensayo } \\
\text { clínico } \\
\text { fase II }\end{array}$ & Alemania & $\begin{array}{l}\mathrm{N}=30 \\
\text { No constan grupos. } \\
\text { Edad media } 60 \\
\text { años } \\
\mathrm{H} / \mathrm{M}=24 / 6\end{array}$ & $\begin{array}{l}\text { Neoplasia } \\
\text { metastásica } \\
\text { de células } \\
\text { renales }\end{array}$ & $\begin{array}{l}\text { Capecitabina en } \\
\text { pacientes con } \\
\text { insuficiencia renal } \\
\text { metastásica. }\end{array}$ & $\begin{array}{l}\text { Ciclos de } 8 \\
\text { semanas de } \\
\text { tratamiento } \\
\text { repetido hasta } \\
\text { por } 3 \text { cursos }\end{array}$ & $\begin{array}{l}\text { La terapia } \\
\text { fue tolerada } \\
\text { correctamente por } \\
\text { los pacientes. La } \\
\text { administración de } \\
\text { la capecitabina } \\
\text { permitió el } \\
\text { tratamiento } \\
\text { ambulatorio o } \\
\text { en el hogar, } \\
\text { reduciendo } \\
\text { gastos y pudiendo } \\
\text { mejorar la calidad } \\
\text { de vida en el } \\
\text { entorno paliativo. }\end{array}$ \\
\hline $\begin{array}{l}\text { Ron, et al. } \\
1996 \text { (21) }\end{array}$ & $\begin{array}{l}\text { Ensayo } \\
\text { clínico } \\
\text { controlado }\end{array}$ & Israel & $\begin{array}{l}\mathrm{N}=61 \\
\text { No constan grupos. } \\
\text { No consta edad } \\
\text { media. } \\
\text { No consta H/M. }\end{array}$ & $\begin{array}{l}\text { Neoplasia } \\
\text { colorrectal } \\
\text { avanzada }\end{array}$ & $\begin{array}{l}\text { Rendimiento } \\
\text { terapéutico, efecto } \\
\text { sobre calidad } \\
\text { vida y rentabilidad } \\
\text { de ftorafur oral } \\
\text { en atención } \\
\text { domiciliaria. }\end{array}$ & $\begin{array}{l}\text { Entre 2-18 } \\
\text { meses (media } \\
\text { de 6,4) }\end{array}$ & $\begin{array}{l}\text { Atención } \\
\text { domiciliaria } 70 \% \\
\text { menos costosa. } \\
\text { Tratamiento en el } \\
\text { hogar con ftorafur } \\
\text { oral fue la opción } \\
\text { más deseable de } \\
\text { estos pacientes. }\end{array}$ \\
\hline
\end{tabular}


Tabla 1. Características de los estudios incluidos en la revisión sobre ventajas de la quimioterapia domiciliaria en los enfermos adultos con neoplasias

\begin{tabular}{|c|c|c|c|c|c|c|c|}
\hline Autor; año & Diseño & País & Población & $\begin{array}{l}\text { Patología } \\
\text { (neoplasia) }\end{array}$ & $\begin{array}{l}\text { Intervención } \\
\text { (tratamiento) }\end{array}$ & $\begin{array}{l}\text { Periodo del } \\
\text { tratamiento }\end{array}$ & $\begin{array}{l}\text { Resultados } \\
\text { obtenidos }\end{array}$ \\
\hline $\begin{array}{l}\text { lacobelli, et } \\
\text { al. } 1995(22)\end{array}$ & $\begin{array}{l}\text { Ensayo } \\
\text { clínico fase I }\end{array}$ & Italia & $\begin{array}{l}\mathrm{N}=18 \\
\text { No constan grupos. } \\
\text { Edad media } 59,6 \\
\text { años } \\
\mathrm{H} / \mathrm{M}=12 / 6\end{array}$ & $\begin{array}{l}\text { Neoplasias } \\
\text { avanzadas }\end{array}$ & $\begin{array}{l}\text { Interferón alfa } \\
\text { administrado a una } \\
\text { tasa modulada de } \\
\text { ritmo circadiano } \\
\text { para evaluar dosis } \\
\text { máxima tolerada } \\
\text { y toxicidad en el } \\
\text { hogar }\end{array}$ & $\begin{array}{l}\text { Ciclo de } 7 \\
\text { días, con } \\
\text { incremento } \\
\text { de dosis en } \\
6 \text { ciclos con } \\
\text { una semana } \\
\text { de descanso } \\
\text { entre ellos. }\end{array}$ & $\begin{array}{l}\text { La programación } \\
\text { circadiana del } \\
\text { IFN-a permitió una } \\
\text { administración } \\
\text { alta con toxicidad } \\
\text { aceptable, } \\
\text { además de } \\
\text { dos respuestas } \\
\text { objetivas en } \\
\text { pacientes en } \\
\text { los que terapias } \\
\text { previas habían } \\
\text { fracasado. No } \\
\text { se mencionan } \\
\text { diferencias entre } \\
\text { el tratamiento en } \\
\text { casa u hospital. }\end{array}$ \\
\hline $\begin{array}{l}\text { Atzpodien, } \\
\text { et al. } \\
1995 \text { (23) }\end{array}$ & $\begin{array}{l}\text { Ensayo } \\
\text { clínico } \\
\text { fase II }\end{array}$ & Alemania & $\begin{array}{l}\mathrm{N}=152 \\
\text { No constan grupos. } \\
\text { Edad media } 58 \\
\text { años } \\
\mathrm{H} / \mathrm{M}=92 / 62\end{array}$ & $\begin{array}{l}\text { Neoplasia } \\
\text { metastásica } \\
\text { progresiva } \\
\text { de células } \\
\text { renales }\end{array}$ & $\begin{array}{l}\text { Tratamiento con } \\
\text { interleuquina } \\
\text { humana } \\
\text { recombinante } \\
2 \text { subcutánea e } \\
\text { Interferón alfa } 2 \\
\text { subcutáneo. }\end{array}$ & 6 semanas & $\begin{array}{l}\text { Toxicidad } \\
\text { sistémica } \\
\text { drásticamente } \\
\text { reducida, al } \\
\text { tiempo que } \\
\text { se confirmó } \\
\text { la eficacia y } \\
\text { tolerabilidad } \\
\text { terapéutica del } \\
\text { esquema de } \\
\text { combinación de } \\
\text { inmunoterapia } \\
\text { SC de dosis baja. } \\
\text { No se mencionan } \\
\text { diferencias entre } \\
\text { el tratamiento en } \\
\text { casa u hospital. }\end{array}$ \\
\hline
\end{tabular}

$\mathrm{H} / \mathrm{M}=$ relación número de hombres / número de mujeres.

IL-2 = Interleuquina-2; IFN-a = Interferón alfa; 5-FU = 5-FLuorouracilo

$\mathrm{Gcp}=$ Grupo tratamiento en casa primero

$\mathrm{Ghp}=$ Grupo tratamiento en hospital primero

$\mathrm{CHOP}=$ Ciclofosfamida, doxorrubicina, vincristina y prednisona.

13cRA = Ácido 13-cis-retinoico 


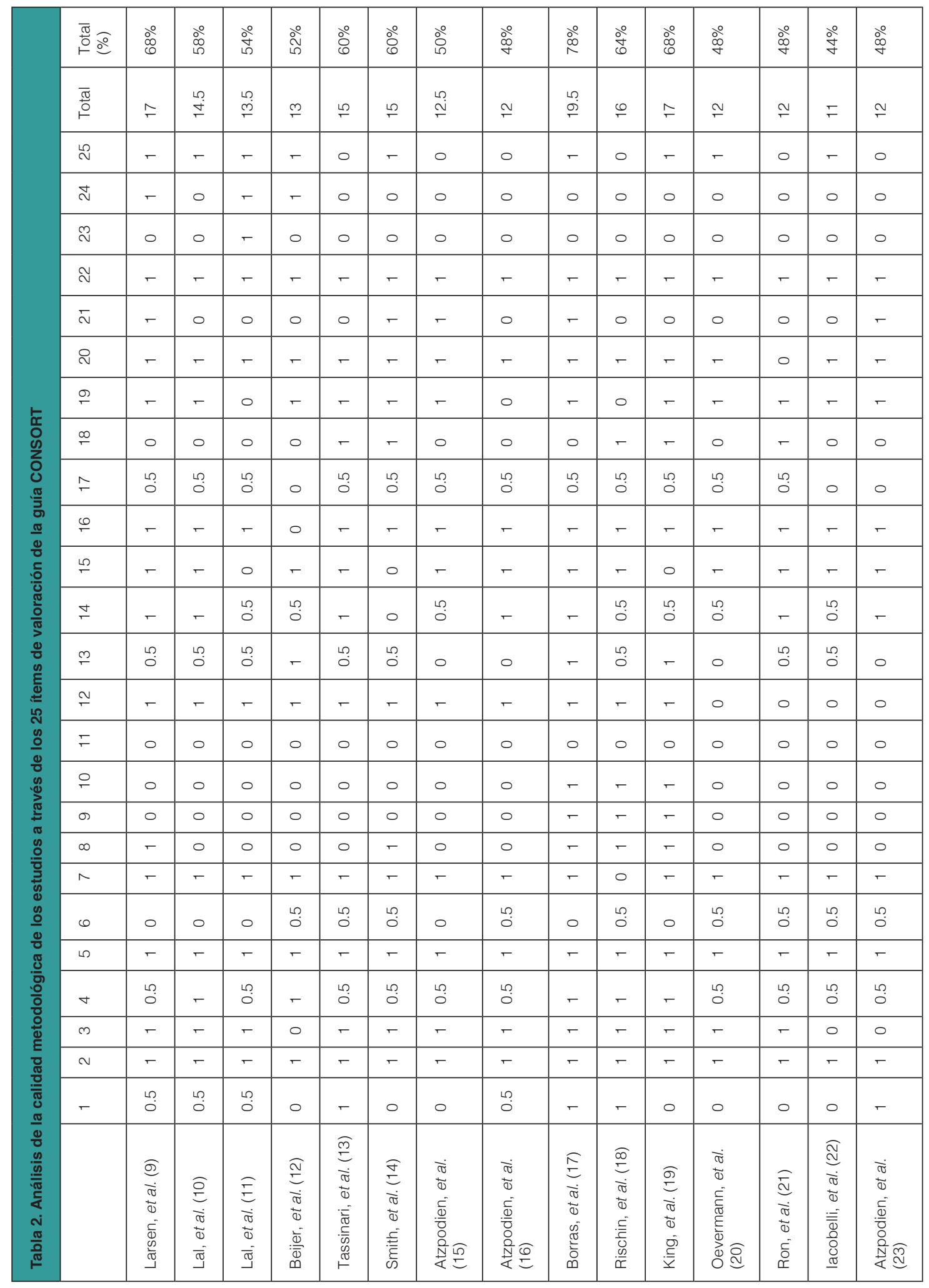




\section{Lugar de la intervención farmacológica (tratamiento quimioterápico)}

La estrategia terapéutica no fue la misma en todos los casos. Los medicamentos más usados fueron el 5-Fluorouracilo y el Interferón-a, los cuales aparecen en 4 (26,7\%) artículos respectivamente: (16-19) y $(15,15,16,22)$. En $7(46,7 \%)$ ensayos se estudió el efecto de un solo fármaco (10-13,20-22), mientras que, en el resto de los artículos se utilizaron al menos 2 quimioterápicos $(9,14-17,19,23)$, alcanzando, en el ensayo de Rischin et al. (18), el número de 9 fármacos quimioterápicos debido a una terapia combinada.

En $4(26,7 \%)$ de los ensayos clínicos revisados se comparaba directamente el tratamiento administrado en casa frente al tratamiento administrado en hospital $(9,14,17,18)$, en los $11(73,3 \%)$ trabajos restantes se estudiaron las respuestas al tratamiento aplicado directamente en casa (1013,15,16,19-23). En uno de ellos (10), la primera dosis del tratamiento se llevó a cabo en el hospital.

\section{Principales resultados}

En 12 (80\%) de los ensayos clínicos revisados se observó que las intervenciones con el tratamiento quimioterápico administrado en el hogar eran seguras, factibles, beneficiosas y/o predominantemente preferidas por los pacientes (9-14,16-21).

En $3(20 \%)$ documentos, no se mencionaron diferencias entre el tratamiento quimioterápico administrado en el hogar o en el hospital $(15,22,23)$.

Por tanto, no hubo ningún estudio donde los pacientes prefirieran en tratamiento hospitalario frente a ser tratados en el domicilio. Ahora bien, en el ensayo de Lal et al. (10), de 2015, se documentó la preferencia de un paciente a ser tratado en el hospital, pero se debió a la aparición de efectos secundarios al tratamiento.

Con respecto al coste del tratamiento se comprobó discrepancias entre algunos trabajos: Lal et al. (11) y King et al. (19), concluyeron que a pesar de que la quimioterapia domiciliaria fuera la opción preferida por los pacientes también demostró ser la más cara. Rischin et al. (18), con una estrategia terapéutica de hasta 9 quimioterápicos, estimaron un aumento de 83 dólares cada tratamiento de quimioterapia en el hogar en comparación con el tratamiento en el hospital. En cambio, Ron et al. (21) afirmaron que el tratamiento domiciliario con ftorafur oral fue hasta un $70 \%$ menos costoso que en el hospital; en línea con lo recogido por Tassinari et al. (13). Ahora bien, según Lal et al. (11), el mayor o menor coste del tratamiento dependió del medicamento y tipo de tratamiento.

En el resto de los ensayos clínicos revisados no se mencionaron diferencias relacionadas con el tratamiento domiciliario versus hospitalario.

\section{DISCUSIÓN}

De los resultados obtenidos en la revisión de los diferentes artículos se pudo comprobar la existencia de estudios que destacaban ventajas del tratamiento quimioterápico domiciliario en los enfermos adultos con neoplasias.

La obsolescencia de los documentos revisados presentó resultados muy similares a los expuestos en un trabajo anterior por Bernabeu-Martínez et al. (24) y de mayor antigüedad a lo previstos en el área de las ciencias de la salud (25). El escaso número de ensayos clínicos encontrados y su baja actualidad mostró la necesidad de la actualización del tema. Ahora bien, la única revisión sistemática, indizada en la base de datos MEDLINE, anterior a este trabajo que versa sobre el tema revisado data del año 2018 (26). Si bien, se localizó un protocolo de revisión sistemática, del año 
2018, sobre los impactos en los resultados de salud y en la utilización de recursos de la administración de quimioterapia parenteral en el hogar, pero sin resultados definitivos (27).

El diseño de los estudios revisados, ensayos clínicos, reforzó la validez de los resultados según el grado de evidencia y recomendación de la U.S. Preventive Services Task Force (USPSTF) $(28,29)$. El ceñir la revisión a ensayos clínicos se debió a la búsqueda de una consistente relación causa-efecto de la administración de quimioterápicos en el domicilio y la percepción del paciente en relación a su satisfacción.

La evaluación de la calidad de los estudios incluidos en este trabajo mediante CONSORT no resultó muy elevada a pesar de tratarse de diseños robustos, de todos modos no se eliminó ningún estudio dado el bajo número de ellos, situación ya vista anteriormente en otras revisiones sistemáticas $(29,30)$. También pudo influir que los artículos publicados antes de 2010, año en el que se publicó la declaración CONSORT, no tuvieran un referente de calidad y por ello presentaran puntuaciones bajas (8).

El idioma de los estudios fue el esperado, estando todos los trabajos escritos en inglés. Este idioma es el elegido para la publicación de la mayoría de los artículos ya que hacerlo en otra lengua puede resultar negativo para la visibilidad, el factor de impacto y las citaciones. Además, el número de revistas anglófonas contenidas en las bases de datos actualmente es muy elevado $(25,29)$.

La población incluida en los estudios presentó edades avanzadas. Esta situación es común en las unidades de $\mathrm{HaD}$ ya que para grupos de población adulta o anciana con patologías crónicas o terminales la $\mathrm{HaD}$ encuentra todo su significado y ha demostrado ser un modelo eficiente. El desarrollo de esquemas de hospitalización domiciliaria integral es una oportunidad como alternativa a la hospitalización convencional adaptada a pacientes ancianos en nuestro entorno asistencial (31).

La patología tratada en todos los trabajos seleccionados fue las neoplasias, en algunos casos, en fase de tratamiento paliativo, probablemente debido a la creciente incidencia de estas enfermedades (29).

En cuanto al período de seguimiento hay estudios que consideran necesario un periodo de varias semanas para poder valorar los resultados (29), condición que se cumplió en la mayoría de los estudios revisados.

Con respecto a las discrepancias observadas con el coste del tratamiento en $\mathrm{HaD}$, además de deberse fundamentalmente a las estrategias farmacológicas, como se vio en los ensayos revisados (13), hay otros estudios que avalan la eficiencia de los tratamientos domiciliarios. El coste por estancia es sensiblemente inferior a la modalidad de la hospitalización convencional. En aquellos enfermos susceptibles de ser atendidos en régimen de $\mathrm{HaD}$, el ahorro se sitúa entre el 15 y el $80 \%$ de los gastos de hospitalización, según centros y patologías (1). Según Caplan et al. (32), aparte de que los resultados en salud de la $\mathrm{HaD}$ son similares e incluso superiores a los de la hospitalización convencional para un grupo seleccionado de pacientes, los costes resultan significativamente menores.

La estrategia terapéutica domiciliaria, en la población a estudio, se reafirmó como adecuada y preferida por los enfermos y sus cuidadores, demostrándose una modalidad satisfactoria por el alto contenido de relación con el enfermo y su entorno que se percibe como un elemento de calidad y atención excelente (1). Y, se manifestó segura para el paciente, en línea con lo expuesto por Estrada Cuxart et al. (1), ya que los indicadores de seguridad clínica del paciente en HaD son equiparables, e incluso mejores que el de las unidades de hospitalización convencional, tendiéndose a evitar sobreindicación de tratamientos y de pruebas diagnósticas. De hecho, en esta revisión solo en el ensayo de Lal et al. (10), se documentó la preferencia de un paciente a ser tratado en el hospital, pero se debió a la aparición de efectos secundarios al tratamiento.

Estos resultados observados, estuvieron en línea a la revisión sistemática publicada por Cool et al.(26), donde exponen que la mayoría de los modelos informados para hospitalización oncológica 
domiciliaria demostraron que esta es una alternativa segura, equivalente y aceptable a la atención hospitalaria ambulatoria. Pero, serían necesarios más ensayos clínicos bien diseñados para evaluar su impacto económico.

Posibles limitaciones al estudio: La principal limitación sería el escaso número de estudios recuperados que impidió tener una clara evidencia entre la intervención estudiada.

Por otro lado, las bases de datos de Scopus y Web of Science recuperaron muchos trabajos que finalmente fueron irrelevantes, lo que podría deberse a la falta de indexación (la búsqueda se realizó en formato de texto consultando el título, el resumen y las palabras clave) y la imposibilidad de limitar la búsqueda por tipo de artículo (restringido a ensayos clínicos). Este alto "ruido" de documentos también se observó en otras recientes revisiones sistemáticas $(33,34)$.

Por todo lo anteriormente expuesto, se podría concluir: La quimioterapia domiciliaria es una alternativa factible y segura para los pacientes, la cual es preferida por estos y sus cuidadores. El mayor o menor coste dependió principalmente del medicamento y tipo de tratamiento.

\section{BIBLIOGRAFÍA}

1. Estrada Cuxart O, Massa Domínguez B, Ponce González MÁ, Mirón Rubio M, Torres Corts A, Mujal Martínez A, et al. Proyecto HAD 2020: una propuesta para consolidar la hospitalización a domicilio en España. Hosp Domic. 2017;1(2):93-117. DOI: 10.22585/hospdomic.v1i2.13

2. Cool L, Missiaen J, Vandijck D, Lefebvre T, Lycke M, De Jonghe PJ, et al. An observational pilot study to evaluate the feasibility and quality of oncological home-hospitalization. Eur J Oncol Nurs. 2019;40:44-52. DOI: 10.1016/j.ejon.2019.03.003; PMID: 31229206

3. Raphaël R, Yves D, Giselle C, Magali M, Odile CM. Cancer treatment at home or in the hospital: what are the costs for French public health insurance? Findings of a comprehensive-cancer centre. Health Policy. 2005;72(2):141-8. DOI: 10.1016/j.healthpol.2004.07.001; PMID: 15802149

4. Corrie PG, Moody M, Wood V, Bavister L, Prevost T, Parker RA, et al. Protocol for the OUTREACH trial: a randomised trial comparing delivery of cancer systemic therapy in three different settings: patient's home, GP surgery and hospital day unit. BMC Cancer. 2011;11:e467. DOI: 10.1186/1471-2407-11-467; PMID: 22035502

5. Ayala de Calvo LE, Sepulveda-Carrillo GJ. Care needs of cancer patients undergoing ambulatory treatment. EGlobal. 2016;16(1):353-68. DOI: 10.6018/eglobal.16.1.231681

6. Massa Domínguez B. La hospitalización a domicilio en el siglo XXI. Hosp Domic. 2017;1(1):7-9. DOI: 10.22585/hospdomic.v111.8

7. Wanden-Berghe C, Sanz-Valero J. Systematic reviews in nutrition: standardized methodology. Br J Nutr. 2012;107(2 Suppl):S3-7. DOI: 10.1017/S0007114512001432; PMID: 22591902

8. Pandis N, Chung B, Scherer RW, Elbourne D, Altman DG. CONSORT 2010 statement: extension checklist for reporting within person randomised trials. BMJ. 2017;357:j2835. DOI: 10.1136/bmj. j2835; PMID: 28667088

9. Larsen FO, Christiansen AB, Rishøj A, Nelausen KM, Nielsen DL. Safety and feasibility of homebased chemotherapy. Dan Med J. 2018;65(5):pii:A5482. PMID: 29726319

10. Lal R, Hillerdal GN, Shah RNH, Crosse B, Thompson J, Nicolson M, et al. Feasibility of home delivery of pemetrexed in patients with advanced non-squamous non-small cell lung cancer. Lung Cancer. 2015;89(2):154-60. DOI: 10.1016/j.lungcan.2015.05.003; PMID: 26101013 
11. Lal R, Bourayou N, Hillerdal G, Nicolson M, Vikstrom A, Lorenzo M, et al. Home administration of maintenance pemetrexed for patients with advanced non-squamous non-small cell lung cancer: rationale, practicalities and phase II feasibility study design. Health Qual Life Outcomes. 2013;11:e163. DOI: 10.1186/1477-7525-11-163; PMID: 24090033

12. Beijer S, Gielisse EAR, Hupperets PS, van den Borne BEEM, van den Beuken-van Everdingen M, Nijziel MR, et al. Intravenous ATP infusions can be safely administered in the home setting: a study in pre-terminal cancer patients. Invest New Drugs. 2007;25(6):571-9. DOI: 10.1007/ s10637-007-9076-1; PMID: 17786387

13. Tassinari D, Poggi B, Nicoletti S, Fantini M, Tamburini E, Possenti C, et al. Zoledronic acid treatment at home: safety data from an observational prospective trial. J Palliat Med. 2007;10(2):352-8. DOI: 10.1089/jpm.2006.0122; PMID: 17472506

14. Smith AG, Soutar RL, Schey S, Andrews CD, Baister ER, Bilbrough C, et al. Home care versus hospital care in patients with multiple myeloma treated with pamidronate. Int J Palliat Nurs. 2004;10(3):144-9. DOI: 10.12968/ijpn.2004.10.3.12602; PMID: 15126959

15. Atzpodien J, Küchler T, Wandert T, Reitz M. Rapid deterioration in quality of life during interleukin-2- and alpha-interferon-based home therapy of renal cell carcinoma is associated with a good outcome. Br J Cancer. 2003;89(1):50-4. DOI: 10.1038/sj.bjc.6600996; PMID: 12838299

16. Atzpodien J, Hoffmann R, Franzke M, Stief C, Wandert T, Reitz M. Thirteen-year, long-term efficacy of interferon 2alpha and interleukin 2-based home therapy in patients with advanced renal cell carcinoma. Cancer. 2002;95(5):1045-50. DOI: 10.1002/cncr.10783; PMID: 12209689

17. Borras JM, Sanchez-Hernandez A, Navarro M, Martinez M, Mendez E, Ponton JL, et al. Compliance, satisfaction, and quality of life of patients with colorectal cancer receiving home chemotherapy or outpatient treatment: a randomised controlled trial. BMJ. 2001;322(7290):e826. DOI: 10.1136/bmj.322.7290.826; PMID: 11290635

18. Rischin D, White MA, Matthews JP, Toner GC, Watty K, Sulkowski AJ, et al. A randomised crossover trial of chemotherapy in the home: patient preferences and cost analysis. Med J Aust. 2000;173(3):125-7. PMID: 10979376

19. King MT, Hall J, Caleo S, Gurney HP, Harnett PR. Home or hospital? An evaluation of the costs, preferences, and outcomes of domiciliary chemotherapy. Int J Health Serv. 2000;30(3):557-79. DOI: 10.2190/CY03-EV15-K38Y-X4AA; PMID: 11109181

20. Oevermann K, Buer J, Hoffmann R, Franzke A, Schrader A, Patzelt T, et al. Capecitabine in the treatment of metastatic renal cell carcinoma. Br J Cancer. 2000;83(5):583-7. DOI: 10.1054/ bjoc.2000.1340; PMID: 10944596

21. Ron IG, Lotan A, Inbar MJ, Chaitchik S. Advanced colorectal carcinoma: redefining the role of oral ftorafur. Anticancer Drugs. 1996;7(6):649-54. DOI: 10.1097/00001813-199608000-00005; PMID: 8913433

22. Iacobelli S, Garufi C, Irtelli L, Martino MT, Santobuono F, Vicario G, et al. A phase I study of recombinant interferon-alpha administered as a seven-day continuous venous infusion at circadian-rhythm modulated rate in patients with cancer. Am J Clin Oncol. 1995;18(1):27-31. DOI: 10.1097/00000421-199502000-00006; PMID: 7847255

23. Atzpodien J, Lopez Hänninen E, Kirchner H, Bodenstein H, Pfreundschuh M, Rebmann U, et al. Multiinstitutional home-therapy trial of recombinant human interleukin-2 and interferon alfa-2 in progressive metastatic renal cell carcinoma. J Clin Oncol. 1995;13(2):497-501. DOI: 10.1200/ JCO.1995.13.2.497; PMID: 7844611 
24. Bernabeu-Martínez MA, Sanz Valero J. Análisis bibliométrico y temático de la producción científica existente en la base de datos bibliográfica MEDLINE sobre medicamentos peligrosos en las Unidades de Hospitalización a Domicilio. Hosp Domic. 2018;2(3):101-15. DOI: 10.22585/ hospdomic.v2i3.50

25. Sanz-Valero J, Wanden-Berghe C. Análisis bibliométrico de la producción científica, indizada en MEDLINE, sobre los servicios de salud proporcionados por las unidades de hospitalización a domicilio. Hosp Domic. 2017;1(1):21-34. DOI: 10.22585/hospdomic.v111.3

26. Cool L, Vandijck D, Debruyne P, Desmedt M, Lefebvre T, Lycke M, et al. Organization, quality and cost of oncological home-hospitalization: A systematic review. Crit Rev Oncol Hematol. 2018;126:145-53. DOI: 10.1016/j.critrevonc.2018.03.011; PMID: 29759557

27. Mittaine-Marzac B, De Stampa M, Bagaragaza E, Ankri J, Aegerter P. Impacts on health outcomes and on resource utilisation of home-based parenteral chemotherapy administration: a systematic review protocol. BMJ Open. 2018;8(5):e020594. DOI: 10.1136/bmjopen-2017-020594; PMID: 29743329

28. Whitlock EP, Orleans CT, Pender N, Allan J. Evaluating primary care behavioral counseling interventions: an evidence-based approach. Am J Prev Med. 2002;22(4):267-84. DOI: 10.1016/ s0749-3797(02)00415-4; PMID: 11988383

29. Aracil-Lavado E, Wanden-Berghe C, Sanz-Valero J. Evaluación de la calidad de vida según el estado nutricional del paciente paliativo adulto: revisión sistemática. Hosp Domic. 2017;1(4):199210. DOI: 10.22585/hospdomic.v1i4.27

30. Domingo Pena V, Sanz Valero J. Efectos de la inmunoterapia en el paciente adulto en el domicilio: revisión sistemática. Hosp Domic. 2018;2(2):15-25. DOI: 10.22585/hospdomic.v2i2.40

31. Mas MÀ, Santaeugènia $S$. Hospitalización domiciliaria en el paciente anciano: revisión de la evidencia y oportunidades de la geriatría. Rev Esp Geriatr Gerontol. 2015;50(1):26-34. DOI: 10.1016/j.regg.2014.04.003

32. Caplan GA, Sulaiman NS, Mangin DA, Aimonino Ricauda N, Wilson AD, Barclay L. A metaanalysis of «hospital in the home». Med J Aust. 2012;197(9):512-9. DOI: 10.5694/mja12.10480; PMID: 23121588

33. Bernabeu-Martínez MA, Ramos Merino M, Santos Gago JM, Álvarez Sabucedo LM, WandenBerghe C, Sanz-Valero J. Guidelines for safe handling of hazardous drugs: A systematic review. PLoS ONE. 2018;13(5):e0197172. DOI: 10.1371/journal.pone.0197172; PMID: 29750798

34. Gea Cabrera A, Sanz-Lorente M, Sanz-Valero J, López-Pintor E. Compliance and Adherence to Enteral Nutrition Treatment in Adults: A Systematic Review. Nutrients. 2019;11(11). DOI: 10.3390/nu11112627; PMID: 31684024 